\section{FATORES DE RISCO PARA DOENÇAS CRÔNICAS NÃO TRANSMISSÍVEIS E VARIÁVEIS SOCIODEMOGRÁFICAS DE SERVIDORES PÚBLICOS}

\author{
Risk Factors for Non-Communicable Diseases and \\ Sociodemographic Variables of Civil Servants \\ Factores de riesgo para enfermedades crónicas no trasmisibles \\ y las variables sociodemográficas de funcionarios
}

\begin{abstract}
RESUMO
Objetivo: Associar fatores de risco para doenças crônicas não transmissíveis (DCNT) com variáveis sociodemográficas de servidores de uma instituição pública de ensino superior. Métodos: Estudo transversal, realizado no período de 2012 a 2013. Utilizou-se um questionário com variáveis sociodemográficas, fatores de risco para DCNT e aferição de peso e estatura. Classificou-se o estado nutricional de acordo com o índice de massa corporal. Analisaram-se os dados pelos programas Epi-Info 3.2.1 e Bioestat 5.0. As associações foram verificadas pelos testes qui-quadrado de Yates, de tendência e exato de Fisher $(\mathrm{p} \leq 0,05)$. Resultados: Participaram 225 servidores, maioria de mulheres (64,4\%), na faixa etária entre 45-54 anos (37,3\%) e escolaridade superior a 12 anos de estudo (85,8\%). O sexo associou-se ao excesso de peso $(\mathrm{p}=0,034)$, consumo diário de leite integral $(\mathrm{p}=0,023)$, consumo insuficiente de frutas, legumes e verduras - FLV ( $p=0,020)$ e consumo insuficiente de feijão $(p=0,000)$, sendo mais frequentes entre mulheres. Entre os homens, foi mais frequente o consumo excessivo de bebida alcoólica $(\mathrm{p}=0,000)$. $\mathrm{O}$ excesso de peso associou-se à faixa etária $(\mathrm{p}=0,008)$. $\mathrm{O}$ hábito de fumar $(\mathrm{p}=0,004)$ e o consumo diário de leite integral $(\mathrm{p}=0,016)$ apresentaram associação com a escolaridade. Conclusão: Encontrou-se elevada prevalência de fatores de risco para doenças crônicas não transmissíveis na amostra estudada, com associação para sexo, faixa etária e escolaridade. $\mathrm{O}$ excesso de peso apresentou maior ocorrência nas mulheres e nas idades acima de 45 anos, hábitos alimentares inadequados no sexo feminino, maior ingestão de bebida alcoólica no masculino e hábito de fumar nos indivíduos com maior escolaridade.
\end{abstract}

Descritores: Fatores de Risco; Doenças Crônicas; Estilo de Vida.

\section{ABSTRACT}

Objective: To associate risk factors for chronic noncommunicable diseases (NCDs) with sociodemographic variables of public servants of a higher education institution. Methods: Cross-sectional study conducted from 2012 to 2013. It was used a questionnaire with sociodemographic variables, risk factors for NCDs and weight and height measurement of respondents. Nutritional status was classified according to the body mass index. Data were analyzed by Epi-Info 3.2.1 and Bioestat 5.0 softwares. Associations were checked by Yates' chi-squared test, trend test and Fisher's exact test ( $p \leq 0.05)$. Results: A total of 225 servants participated in the study, most being women (64.4\%), aged between 45-54 years (37.3\%), and with more than 12 years of education (85.8\%). The gender was associated with excess weight $(p=0.034)$, daily consumption of whole milk $(p=0.023)$, insufficient intake of fruits and vegetables $(p=0.020)$ and insufficient intake of beans $(p=0.000)$, being more frequent among women. As to men, the excessive consumption of alcoholic beverages $(p=0.000)$ was more frequent. Excess weight was associated with age $(p=0.008)$. Smoking $(p=0.004)$ and daily consumption of whole milk $(p=0.016)$ were associated with education. Conclusion: There was high prevalence of risk factors for chronic noncommunicable diseases in the study sample, being associated with gender, age and education. Excess weight presented greater occurrence among women and in individuals aged over 45 years, women's inadequate eating habits, higher consumption of alcohol among men and smoking habit in individuals with higher education levels.

Descriptors: Risk Factors; Chronic Diseases; Lifestyle.
Artigo Original

Luciana Soares do Nascimento ${ }^{(1)}$

Rosa Maria Dias ${ }^{(1)}$

Cláudia Daniele Tavares Dutra ${ }^{(1)}$ Luísa Margareth Carneiro da Silva ${ }^{(1)}$

Luiza Né Pedrosa ${ }^{(1)}$ Josiana Sampaio Araújo ${ }^{(1)}$ Andréa das Graças Ferreira Frazão $^{(1)}$

1) Universidade Federal do Pará - UFPA Belém (PA) - Brasil

Recebido em: 17/05/2015 Revisado em: 22/05/2015 Aceito em: 10/06/2015 


\section{RESUMEN}

Objetivo: Asociar los factores de riesgo para enfermedades crónicas no trasmisibles (ECNT) y las variables sociodemográficas de los funcionarios de una institución pública de enseñanza superior. Métodos: Estudio transversal realizado entre el periodo de 2012 y 2013. Se utilizó un cuestionario con las variables sociodemográficas, los factores de riesgo para las ECNT y la evaluación del peso y la estatura de los entrevistados. Se clasificó el estado nutricional según el índice de masa corporal. Los datos fueron analizados con los Programas Epi-Info 3.2.1 y el Bioestat 5.0. Las asociaciones fueron verificadas a través de las pruebas de Chi-cuadrado de Yates, de tendencia y el test exacto de Fisher $(p \leq 0,05)$. Resultados: Participaron 225 funcionarios en su mayoría mujeres $(64,4 \%)$ en la franja de edad entre 45-54 años (37,3\%) y más de 12 años de estudio (85,8\%). El sexo se asoció con el exceso de peso $(p=0,034)$, el consumo diario de la leche integral ( $p=0,023)$, el consumo insuficiente de frutas, legumbres $y$ verduras $-F L V(p=0,020)$ y el consumo insuficiente de frijoles ( $p=0,000)$, más frecuentes en las mujeres. El consumo excesivo de bebida alcohólica fue más frecuente entre los hombres $(p=0,000)$. El exceso de peso se asoció con la franja de edad ( $p=0,008)$. La costumbre de fumar $(p=0,004)$ y el consumo diario de la leche integral $(p=0,016)$ se asociaron con la escolaridad. Conclusión: Se encontró elevada prevalencia de los factores de riesgo para las enfermedades crónicas no trasmisibles en la muestra investigada y su asociación con el peso, la franja de edad y la escolaridad. El exceso de peso se dio más en las mujeres de más de 45 años, con hábitos alimentarios inadecuados para el sexo femenino, mayor ingestión de bebida alcohólica en los hombres y la costumbre de fumar en los individuos con más escolaridad.

Descriptores: Factores de Riesgo; Enfermedad Crónica; Estilo de Vida.

\section{INTRODUÇÃO}

Nas últimas décadas, são observadas no Brasil diversas transformações sociodemográficas e econômicas que ocasionaram relevantes mudanças no estilo de vida da população e convergiram para uma dieta mais rica em gorduras, açúcares, alimentos refinados, reduzida em carboidratos complexos e fibras, como também a um declínio progressivo da atividade física e aumento do etilismo e tabagismo. Esses fatores, associados, contribuíram para modificações relevantes no perfil nutricional, epidemiológico e no padrão de morbimortalidade da população, com o aumento na prevalência de doenças crônicas não transmissíveis (DCNT) ${ }^{(1-3)}$.

As DCNT são responsáveis pelas maiores taxas de morbimortalidade no país e pela maior proporção das despesas com assistências ambulatorial e hospitalar ${ }^{(4)}$.

De acordo com a World Health Organization $(\mathrm{WHO})^{(5)}$, um dos principais fatores de risco para o desenvolvimento de DCNT é o excesso de peso, que pode ser proveniente de hábitos de vida não saudáveis, como alimentação inadequada e atividade física insuficiente.

Segundo estimativas da Pesquisa de Orçamentos Familiares (2008-2009)(6), as prevalências de excesso de peso e obesidade em adultos no Brasil vêm crescendo: cerca de $49 \%$ da população adulta está com excesso de peso e $15 \%$ estão na faixa de obesidade.

O aumento da prevalência de indivíduos com excesso de peso aumenta também a frequência de pessoas com uma ou mais condições comórbidas, como hipertensão arterial, diabetes tipo 2, doenças cardiovasculares, entre outras doenças crônicas, debilitantes e de alto custo social, que acabam por elevar consequentemente as taxas de mortalidade $^{(7)}$.

A promoção da saúde capacita o indivíduo e a sociedade para atuarem na melhoria da qualidade de vida e saúde, incluindo uma maior participação no controle do processo para obtenção de um completo estado de bem-estar físico, mental e social, que possibilite identificar as aspirações e necessidades, e assim ter a possibilidade de modificar favoravelmente o estilo de vida e ambiente ${ }^{(8)}$.

Analisar hábitos de vida relacionados à saúde de diferentes populações pode trazer valiosas contribuições para o entendimento da prevalência de doenças relacionadas ao estilo de vida, principalmente as $\mathrm{DCNT}^{(9)}$. Assim, o conhecimento da distribuição dos fatores de risco e protetores é fundamental para atuar sobre o processo saúde-doença, com vistas ao desenvolvimento de políticas públicas específicas para a melhoria da qualidade de vida da população, permitindo ainda o monitoramento dessas ações ${ }^{(10)}$.

Nas instituições públicas de ensino superior existem variados tipos de ocupações, como funções administrativas e docência, que são pouco estudadas, especialmente quanto ao estado nutricional e hábito de vida desses servidores. Nesse contexto, o presente estudo teve por objetivo associar fatores de risco para doenças crônicas não transmissíveis (DCNT) com variáveis sociodemográficas de servidores de uma instituição pública de ensino superior.

\section{MÉTODOS}

$\mathrm{O}$ estudo foi de delineamento transversal, descritivo e analítico, realizado no período de março de 2012 a novembro de 2013. A amostra foi por conveniência, abrangendo técnico-administrativos e docentes, independentemente do sexo, lotados nos diversos institutos da Universidade Federal do Pará, Campus Belém, Brasil.

Incluíram-se servidores no exercício da função, sem limitações físicas que impedissem a tomada das medidas antropométricas e preenchimento do formulário de pesquisa, 
sendo excluídos gestantes e portadores de necessidades especiais.

Os dados foram coletados por meio de entrevistas, utilizando-se de formulário próprio, elaborado segundo as variáveis do estudo.

Realizou-se a avaliação antropométrica, cujas medidas de peso e estatura foram coletadas de acordo com procedimentos descritos no Anthropometric Standardization Reference Manual $^{(11)}$ e orientações do Ministério da Saúde ${ }^{(12)}$. Mensurou-se o peso em duas tomadas, com uso de balança digital da marca Seca ${ }^{\circledR}$, com capacidade para $180 \mathrm{~kg}$ e precisão de $100 \mathrm{~g}$. Para a obtenção da medida de estatura, utilizou-se o estadiômetro da marca Alturexata ${ }^{\circledR}$, com precisão de $1 \mathrm{~mm}$, em duas tomadas. Aplicaram-se as medidas de peso e estatura para o cálculo do índice de massa corporal (IMC), em que o peso em quilogramas foi dividido pelo quadrado da estatura em metros.

Coletaram-se variáveis sociodemográficas (sexo, idade, escolaridade, estado civil, cor/raça autorreferida e função). As variáveis relacionadas aos fatores de risco foram: 1) excesso de peso (IMC, considerado risco a partir de $\left.\geq 25 \mathrm{Kg} / \mathrm{m}^{2}\right)^{(13)}$, incluindo os diagnósticos de préobesidade e obesidade; 2) hábito de fumar; 3 ) consumo de bebida alcoólica, considerado abusivo a partir do consumo superior a quatro doses para mulheres e cinco doses para homens, numa mesma ocasião, pelo menos uma vez nos últimos trinta dias, considerando-se como dose de bebida alcoólica uma dose de bebida destilada, uma lata de cerveja ou uma taça de vinho $^{(2,14)}$;4) atividade física, avaliada segundo a categorização preconizada pela $\mathrm{WHO}^{(15)}$, sendo considerados insuficiente ativos no lazer aqueles que se enquadravam nos seguintes critérios: prática de atividade física inferior a 150 minutos semanais, de intensidade leve ou moderada, ou inferior a 75 minutos semanais de atividade física de intensidade vigorosa no lazer; e 5) consumo alimentar, considerado como fator de risco a partir das seguintes situações: consumo de frutas, verduras e legumes $(F L V) \leq$ cinco vezes na semana, consumo de feijão $\leq$ cinco vezes na semana, consumo de refrigerante $\geq$ cinco dias na semana, hábito de consumo de carne com gordura aparente e ingestão de leite integral diariamente ${ }^{(2,14)}$.

Ainda foram considerados fatores de risco o diagnóstico prévio das seguintes morbidades autorreferidas: hipertensão arterial, diabetes, dislipidemias e doenças cardiovasculares $^{(2,14)}$.

Realizou-se análise descritiva e inferencial pelos Programas Epi Info ${ }^{(16)}$ versão 3.2.1 e Bioestat ${ }^{(17)}$ versão 5.0. Para verificar associações entre variáveis sociodemográficas e variáveis consideradas como fatores de risco, utilizaramse os testes qui-quadrado corrigido de Yates, teste exato de Fisher e teste qui-quadrado de tendência, com nível de significância de $5 \%(\mathrm{p} \leq 0,05)$.

O estudo respeitou a Resolução 466/12, sendo aprovado pelo Comitê de Ética em Pesquisa em Seres Humanos, do Instituto de Ciências da Saúde da Universidade Federal do Pará - CEP-ICS/UFPA, sob parecer nº. 262/11.

\section{RESULTADOS}

Participaram da pesquisa 225 servidores, sendo 162 (72\%) técnico-administrativos e $63(28 \%)$ docentes. A maioria era composta por mulheres $(64,4 \% ; n=145)$, na faixa etária entre $45-54$ anos $(37,3 \% ; n=84)$, com média de idade de 47 anos ( $\pm 11,3$ anos), de cor autorreferida parda $(63,6 \% ; n=143)$ e escolaridade superior a 12 anos de estudo $(85,8 \% ; n=193)$. A maior parte dos servidores entrevistados declarou-se casado ou em união estável $(54,7 \% ; n=123)$ (Tabela I).

$\mathrm{Na}$ amostra geral de servidores, os fatores de risco mais frequentes foram consumo insuficiente de FLV (65,8\%; $\mathrm{n}=148)$, excesso de peso $(64,0 \% ; \mathrm{n}=144)$, consumo diário de leite integral $(61,3 \% ; n=138)$, prática insuficiente de atividade física e consumo insuficiente de feijão, ambos com $(55,1 \% ; n=124)$. O consumo excessivo de bebida alcoólica foi referido por $29,8 \%(n=67)$. Dentre as morbidades referidas, a mais frequente foi a dislipidemia $(29,8 \% ; n=67)$, seguida da hipertensão arterial $(25,9 \%$; $=58)$.

Realizou-se associação entre os fatores de risco e o gênero (Tabela II). Os fatores de risco que se associaram com o gênero foram o excesso de peso, com 59,0\% ( $\mathrm{p}=0,034)$, o consumo diário de leite integral $59,4 \%(\mathrm{p}=0,023)$, o consumo insuficiente de FLV, $58,8 \%(\mathrm{p}=0,020)$, e o consumo de feijão menor que cinco dias na semana, 76,8\% $(\mathrm{p}=0,000)$, com maior prevalência entre as mulheres. $\mathrm{O}$ consumo excessivo de bebidas alcoólicas também se associou com o gênero, sendo mais frequente entre os homens, 59,7\% $(\mathrm{p}=0,000)$.

A Tabela III mostra a associação entre os fatores de risco para DCNT e a faixa etária. Nesse aspecto, evidenciou-se que os fatores de risco foram mais frequentes nos servidores pertencentes à faixa etária de 45 a 54 anos, exceto as morbidades autorreferidas diabetes e doenças cardiovasculares, que foram mais recorrentes na faixa etária de 55 a 64 anos $(54,5 \%$ e $42,9 \%$, respectivamente). $\mathrm{O}$ excesso de peso apresentou tendência crescente até os 54 anos, decaindo nas faixas etárias seguintes $(\mathrm{p}=0,008)$.

$\mathrm{Na}$ Tabela IV, estão descritos os resultados da associação entre a escolaridade e os fatores de risco. Apenas o hábito de fumar $(\mathrm{p}=0,004)$ e o consumo diário de leite integral $(\mathrm{p}=0,016)$ associaram-se com a maior escolaridade (em anos de estudo). 
Tabela I - Distribuição dos servidores por sexo segundo variáveis sociodemográficas. Belém-PA, 2012-2013.

\begin{tabular}{|c|c|c|c|c|c|c|}
\hline \multirow{3}{*}{ Variáveis Sociodemográficas } & \multirow{2}{*}{\multicolumn{2}{|c|}{ Amostra }} & \multicolumn{4}{|c|}{ Sexo } \\
\hline & & & \multicolumn{2}{|c|}{ Homens $(\mathbf{n}=\mathbf{8 0})$} & \multicolumn{2}{|c|}{ Mulheres $(n=145)$} \\
\hline & $\mathrm{n}$ & $\%$ & $\mathbf{n}$ & $\%$ & $\mathbf{n}$ & $\%$ \\
\hline \multicolumn{7}{|l|}{ Faixa etária } \\
\hline 18 a 24 & 09 & 4,0 & 03 & 3,8 & 06 & 4,1 \\
\hline 25 a 34 & 33 & 14,7 & 14 & 17,5 & 19 & 13,1 \\
\hline 35 a 44 & 34 & 15,1 & 13 & 16,3 & 21 & 14,5 \\
\hline 45 a 54 & 84 & 37,3 & 27 & 33,8 & 57 & 39,3 \\
\hline 55 a 64 & 61 & 27,1 & 21 & 26,3 & 40 & 27,6 \\
\hline$\geq 65$ & 04 & 1,8 & 02 & 2,5 & 02 & 1,4 \\
\hline \multicolumn{7}{|l|}{ Estado civil } \\
\hline Casado/união estável & 123 & 54,7 & 52 & 65,0 & 71 & 49,0 \\
\hline Solteiro & 63 & 28,0 & 17 & 21,3 & 46 & 31,7 \\
\hline Viúvo/separado & 39 & 17,3 & 11 & 13,8 & 28 & 19,3 \\
\hline \multicolumn{7}{|l|}{ Anos de estudo } \\
\hline 9 a 11 & 32 & 14,2 & 12 & 15,0 & 20 & 13,8 \\
\hline 12 ou mais & 193 & 85,8 & 68 & 85,0 & 125 & 86,2 \\
\hline \multicolumn{7}{|l|}{ Cor/raça autorreferida } \\
\hline Amarela & 05 & 2,2 & 01 & 1,3 & 04 & 2,8 \\
\hline Branca & 59 & 26,2 & 20 & 25,0 & 39 & 26,9 \\
\hline Negra & 18 & 8,0 & 08 & 10,0 & 10 & 6,9 \\
\hline Parda & 143 & 63,6 & 51 & 63,4 & 92 & 63,8 \\
\hline \multicolumn{7}{|l|}{ Função } \\
\hline Docente & 63 & 28,0 & 21 & 26,3 & 42 & 29,0 \\
\hline Técnico administrativo & 162 & 72,0 & 59 & 73,8 & 103 & 71,0 \\
\hline
\end{tabular}

Tabela II - Distribuição de servidores segundo o gênero associado aos fatores de risco para Doenças Crônicas Não Transmissíveis (DCNT). Belém-PA, 2012- 2013.

\begin{tabular}{|c|c|c|c|c|c|}
\hline \multirow[b]{2}{*}{ Fatores de risco para DCNT } & \multicolumn{2}{|c|}{ Amostra } & \multicolumn{2}{|c|}{ Sexo } & \multirow[b]{2}{*}{$p$} \\
\hline & n & $\%$ & $\begin{array}{c}\text { Homens } \\
(\%)\end{array}$ & $\begin{array}{c}\text { Mulheres } \\
\text { (\%) }\end{array}$ & \\
\hline Excesso de peso & 144 & 64,0 & 41,0 & 59,0 & $0,034^{\mathrm{a}}$ \\
\hline Hábito de fumar & 13 & 5,8 & 46,2 & 53,8 & $0,294^{b}$ \\
\hline Consumo de bebida alcoólica & 67 & 29,8 & 59,7 & 40,3 & $0,000^{\mathrm{a}}$ \\
\hline Atividade física insuficiente* & 124 & 55,1 & 30,6 & 69,4 & $0,117^{\mathrm{a}}$ \\
\hline Consumo insuficiente de FLV $(\leq 5 \mathrm{x} /$ semana $)$ & 148 & 65,8 & 41,2 & 58,8 & $0,020^{\mathrm{a}}$ \\
\hline Consumo diário de leite integral & 138 & 61,3 & 40,6 & 59,4 & $0,023^{\mathrm{b}}$ \\
\hline Consumo de carnes com gordura & 55 & 24,6 & 41,8 & 58,2 & $0,313^{\mathrm{a}}$ \\
\hline Consumo de feijão $(\leq 5 \mathrm{x} /$ semana $)$ & 124 & 55,1 & 23,2 & 76,8 & $0,000^{\mathrm{a}}$ \\
\hline Consumo de refrigerante ( $\geq 5 \mathrm{x} /$ semana) & 22 & 9,8 & 50,0 & 50,0 & $0,209^{\mathrm{a}}$ \\
\hline Hipertensão arterial** & 58 & 25,9 & 36,2 & 63,8 & $0,945^{\mathrm{a}}$ \\
\hline Diabetes** & 11 & 4,9 & 18,2 & 81,8 & $0,362^{\mathrm{b}}$ \\
\hline Dislipidemias** & 67 & 29,8 & 28,4 & 71,6 & $0,188^{\mathrm{a}}$ \\
\hline Doenças cardiovasculares** & 14 & 6,2 & 50,0 & 50,0 & $0,188^{\mathrm{b}}$ \\
\hline
\end{tabular}

${ }^{\text {a }}$ Teste qui-quadrado corrigido de Yates; ${ }^{\mathrm{b}}$ Teste Exato de Fisher

IMC - Índice de massa corporal; FLV - Frutas, Legumes e Verduras > quatro doses para mulheres e cinco doses para homens, numa mesma ocasião pelo menos uma vez nos últimos trinta dias; $*<150$ minutos/semana em intensidade moderada ou $<75$ minutos/semana em intensidade vigorosa no lazer; **Morbidades autorreferida 
Tabela III - Distribuição de servidores segundo a faixa etária associada a fatores de risco para Doenças Crônicas Não Transmissíveis (DCNT). Belém-PA, 2012-2013.

\begin{tabular}{|c|c|c|c|c|c|c|c|c|c|}
\hline \multirow[b]{2}{*}{ Fatores de risco para DCNT } & \multicolumn{2}{|c|}{ Amostra } & \multicolumn{6}{|c|}{ Faixa etária } & \multirow[b]{2}{*}{$p$} \\
\hline & $\mathbf{n}$ & $\%$ & $\begin{array}{c}18-24 \\
(\%)\end{array}$ & $\begin{array}{c}25-34 \\
(\%)\end{array}$ & $\begin{array}{c}35-44 \\
(\%)\end{array}$ & $\begin{array}{c}45-54 \\
(\%)\end{array}$ & $\begin{array}{c}55-64 \\
(\%)\end{array}$ & $\begin{array}{l}>65 \\
(\%)\end{array}$ & \\
\hline Excesso de peso $\left(\mathrm{IMC} \geq 25 \mathrm{~kg} / \mathrm{m}^{2}\right)$ & 144 & 64,0 & 0,7 & 13,9 & 14,6 & 38,9 & 29,9 & 2,1 & $0,008^{\mathrm{a}}$ \\
\hline Hábito de fumar & 13 & 5,8 & - & - & - & 61,5 & 38,5 & - & - \\
\hline Consumo de bebida alcoólica & 67 & 29,8 & 7,5 & 11,9 & 11,9 & 40,3 & 26,9 & 1,5 & $0,829^{\mathrm{a}}$ \\
\hline Atividade física insuficiente* & 124 & 55,1 & 4,0 & 13,7 & 12,1 & 41,9 & 27,4 & 0,8 & $0,650^{\mathrm{a}}$ \\
\hline $\begin{array}{l}\text { Consumo insuficiente de FLV } \\
(<5 \mathrm{x} / \text { semana })\end{array}$ & 148 & 65,8 & 4,1 & 15,1 & 11,5 & 37,8 & 29,7 & 1,4 & $0,536^{\mathrm{a}}$ \\
\hline Consumo diário de leite integral & 138 & 61,3 & 3,2 & 14,4 & 12,0 & 38,4 & 28,8 & 3,2 & $0,385^{\mathrm{a}}$ \\
\hline Consumo de carnes com gordura & 55 & 24,6 & 5,5 & 12,7 & 14,5 & 36,4 & 27,3 & 3,6 & $0,791^{\mathrm{a}}$ \\
\hline Consumo de feijão ( $\leq 5 \mathrm{x} /$ semana $)$ & 124 & 55,1 & 1,6 & 14,4 & 16,8 & 43,2 & 24,0 & - & - \\
\hline $\begin{array}{l}\text { Consumo de refrigerante } \\
(\geq 5 \mathrm{x} / \mathrm{semana})\end{array}$ & 22 & 9,8 & 4,3 & 26,1 & 13,0 & 26,1 & 30,4 & - & - \\
\hline Hipertensão arterial** & 58 & 25,8 & - & 8,6 & 6,9 & 43,1 & 37,9 & 3,4 & - \\
\hline Diabetes** & 11 & 4,9 & - & - & 18,2 & 18,2 & 54,5 & 9,1 & - \\
\hline Dislipidemias** & 67 & 29,8 & - & 7,5 & 14,9 & 43,3 & 32,8 & 1,5 & - \\
\hline Doenças cardiovasculares** & 14 & 6,2 & - & 14,3 & 7,1 & 28,6 & 42,9 & 7,1 & - \\
\hline
\end{tabular}

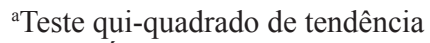

IMC - Índice de massa corporal; FLV - Frutas, Legumes e Verduras > quatro doses para mulheres e cinco doses para homens, numa mesma ocasião pelo menos uma vez nos últimos trinta dias; $*<150$ minutos/semana em intensidade moderada ou $<75$ minutos/semana em intensidade vigorosa no lazer; **Morbidades autorreferidas.

Tabela IV - Distribuição de servidores segundo anos de estudo associados a fatores de risco para Doenças Crônicas Não Transmissíveis. Belém-PA, 2012-2013.

\begin{tabular}{|c|c|c|c|c|c|}
\hline \multirow[b]{2}{*}{ Fatores de risco para DCNT } & \multicolumn{2}{|c|}{ Amostra } & \multicolumn{2}{|c|}{ Anos de Estudo } & \multirow[b]{2}{*}{$p$} \\
\hline & n & $\%$ & $\begin{array}{c}9 \text { a 11anos } \\
(\%)\end{array}$ & $\begin{array}{c}\geq 12 \text { anos } \\
(\%)\end{array}$ & \\
\hline Excesso de peso $\left(\mathrm{IMC} \geq 25 \mathrm{~kg} / \mathrm{m}^{2}\right)$ & 144 & 64,0 & 17,4 & 82,6 & $0,143^{\mathrm{a}}$ \\
\hline Hábito de fumar & 13 & 5,8 & 46,2 & 53,8 & $0,004^{\mathrm{a}}$ \\
\hline Consumo de bebida alcoólica & 67 & 29,8 & 19,4 & 80,6 & $0,214^{\mathrm{a}}$ \\
\hline Atividade física insuficiente* & 124 & 55,1 & 16,1 & 83,9 & $0,474^{\mathrm{a}}$ \\
\hline Consumo insuficiente de FLV $(\leq 5 \mathrm{x} /$ semana $)$ & 148 & 65,8 & 16,9 & 83,1 & $0,165^{\mathrm{a}}$ \\
\hline Consumo diário de leite integral & 138 & 61,9 & 19,2 & 80,8 & $0,016^{\mathrm{a}}$ \\
\hline Consumo de carnes com gordura & 55 & 24,6 & 16,4 & 83,6 & $0,775^{\mathrm{a}}$ \\
\hline Consumo de feijão $(\leq 5 \mathrm{x} /$ semana $)$ & 124 & 55,1 & 16,0 & 84,0 & $0,508^{\mathrm{a}}$ \\
\hline Consumo de refrigerante ( $\geq 5 \mathrm{x} /$ semana $)$ & 22 & 9,8 & 21,7 & 78,3 & $0,212^{\mathrm{a}}$ \\
\hline Hipertensão arterial** & 58 & 25,8 & 10,3 & 89,7 & $0,445^{\mathrm{a}}$ \\
\hline Diabetes** & 11 & 4,9 & 18,2 & 81,8 & $0,481^{\mathrm{a}}$ \\
\hline Dislipidemias $* *$ & 67 & 29,8 & 14,9 & 85,1 & $0,990^{\mathrm{a}}$ \\
\hline Doenças cardiovasculares $* *$ & 14 & 6,2 & 7,1 & 92,9 & $0,379^{\mathrm{a}}$ \\
\hline
\end{tabular}

a Teste Qui-quadrado corrigido de Yates;

IMC - Índice de massa corporal; FLV - Frutas, Legumes e Verduras > quatro doses para mulheres e cinco doses para homens, numa mesma ocasião pelo menos uma vez nos últimos trinta dias; $* 150$ minutos/semana em intensidade moderada ou $<75$ minutos/semana em intensidade vigorosa no lazer;**Morbidades autorreferidas 


\section{DISCUSSÃO}

No presente estudo, foram observadas elevadas frequências de fatores de risco, como excesso de peso, prática insuficiente de atividade física, consumo de bebidas alcoólicas e consumo alimentar inadequado, evidenciando que os servidores estudados seguem tendências nacionais encontradas em pesquisas sobre fatores de risco para $\operatorname{DCNT}^{(6,10,14)}$.

Os efeitos negativos da globalização, da urbanização rápida, da vida sedentária, da alimentação com alto teor calórico e do marketing que estimula o uso de tabaco e álcool são os fatores que refletem no aumento da carga de DCNT no Brasil ${ }^{(18)}$.

O excesso de peso é o quinto principal fator de risco para DCNT que mais leva à morte em todo o mundo. Anualmente, cerca de 2,8 milhões de adultos vêm a óbito devido a fatores ligados ao excesso de peso ${ }^{(4)}$. Além disso, $44 \%$ da carga total de casos de diabetes, $23 \%$ de doença isquêmica do coração e entre $7 \%$ e $41 \%$ da carga de alguns tipos de câncer são atribuídos ao excesso de peso ${ }^{(19)}$.

No presente estudo, a frequência de excesso de peso abrangeu mais da metade da amostra e tendeu a aumentar com a idade, até os 54 anos, e com o aumento da escolaridade, sendo proporcionalmente maior entre as mulheres. Esse grupo também foi mais frequentemente exposto a fatores de risco que desencadeiam tal condição, como atividade física insuficiente e alimentação inadequada.

Esse resultado se assemelha aos encontrados em pesquisas realizadas com adultos na cidade de Salvador$\mathrm{BA}^{(20)} \mathrm{e}$ na cidade de Rio Branco-AC ${ }^{(21)}$, onde a prevalência de excesso de peso foi maior entre mulheres. Porém, difere da tendência brasileira de prevalência de excesso de peso $^{(14)}$, como também dos resultados achados entre servidores de outras instituições de ensino e pesquisa, cuja frequência de excesso de peso foi maior entre os homens e entre indivíduos com menor escolaridade ${ }^{(7,19)}$. Todavia, corrobora com os resultados de uma pesquisa nacional sobre excesso de peso associado à faixa etária, os quais também mostraram uma tendência crescente de excesso de peso até os 54 anos ${ }^{(14)}$.

O consumo abusivo de álcool é outro fator de risco que traz inúmeras consequências para a saúde e qualidade de vida, aumentando a frequência de morbidades que causam morte ou limitações funcionais ${ }^{(22)}$. No presente estudo, a frequência de consumo de bebidas alcoólicas foi de $29,8 \%$. Os resultados deste estudo indicam que os homens constituem o grupo com maior frequência de consumo abusivo de álcool, portanto, são os mais suscetíveis aos seus efeitos adversos.

Esses resultados concordam com os dados do primeiro levantamento nacional sobre o consumo de álcool no Brasil, cujo percentual de brasileiros que referem ter o hábito de consumir regularmente bebida alcoólica varia de $32,4 \%$ a $58,6 \%$, sendo essa prática maior entre os homens ${ }^{(22)}$.

Uma pesquisa nacional mostrou haver relação positiva entre a maior escolaridade e o maior consumo de bebidas alcoólicas, semelhante aos achados neste estudo, em que a maior frequência de consumo de bebida alcoólica foi entre os servidores com 12 anos ou mais de estudo ${ }^{(14)}$.

Estimativas mostram que a alta ingestão de álcool é responsável por $4 \%$ de toda a morbidade e mortalidade ocorridas mundialmente, e esse valor tende a ser crescente ${ }^{(23)}$.

O consumo de tabaco também se destaca como um dos principais fatores de risco das DCNT, e estimativas da Organização Mundial da Saúde (OMS) ${ }^{(5)}$ apontam cerca de seis milhões de mortes anuais decorrentes do uso direto de tabaco ou pelo fumo passivo. No Brasil, um estudo recente sobre carga das doenças relacionadas ao tabaco apontou que aproximadamente $13 \%$ das mortes, em especial as relacionadas às DCNT, são atribuídas ao tabaco, gerando um custo anual de cerca de R $\$ 21$ bilhões ao Sistema Único de Saúde (SUS) ${ }^{(24)}$.

A prevalência de tabagismo no Brasil atinge cerca de $12,1 \%$, sendo os homens maiores consumidores de tabaco do que as mulheres ${ }^{(14)}$. Fato que foi contrário ao encontrado no estudo em questão, no qual o hábito de fumar obteve percentual de 5,8\% e foi mais frequente entre as mulheres.

Esses resultados se assemelham aos encontrados entre servidores da UNICAMP $(6,8 \%)^{(25)}$, porém, muito abaixo do encontrado entre os servidores da Universidade de Brasília - UNB $(19,7 \%)^{(26)}$.

Em ambos os gêneros, a frequência de fumantes tendeu a ser menor antes dos 25 anos de idade ou após os 65 anos. A diminuição da frequência de tabagistas é esperada nas faixas etárias mais elevadas, uma vez que aumenta a cessação do hábito de fumar pela preocupação com a saúde ou devido à presença de doenças ${ }^{(27)}$.

A frequência do hábito de fumar foi maior entre servidores com 12 anos ou mais de estudo, o que difere da pesquisa nacional realizada em 2012, na qual a maioria dos adultos fumantes tinha até oito anos de estudo ${ }^{(14)}$.

Juntamente com o etilismo e o tabagismo, a prática insuficiente de atividade física também é considerada fator de risco relevante para o surgimento de DCNT, já que o sedentarismo está ligado ao aumento dos índices de excesso de peso, podendo levar a outras comorbidades ${ }^{(4)}$.

No presente estudo, a maioria dos servidores referiu prática insuficiente de atividade física $(55,1 \%)$, sendo também mais prevalente entre as mulheres. Essa prática insuficiente tendeu a aumentar com a idade, até os 54 anos, semelhante à tendência observada em pesquisa nacional ${ }^{(14)}$. 
A prática regular da atividade física é componente fundamental para o desenvolvimento de aspectos positivos relacionados à saúde, como na prevenção e no tratamento de síndrome metabólica e de doenças cardiovasculares, devido à consequente redução da massa corporal e dos níveis de pressão arterial e resistência à insulina. Porém, ainda se observa que poucos indivíduos adultos no Brasil são fisicamente ativos ${ }^{(28)}$.

Em estudo realizado com adultos na cidade de Pelotas$\mathrm{RS}^{(29)}$, a prática insuficiente de atividade física entre os entrevistados foi de 75,6\%, sendo mais comum entre as mulheres. Esse resultado é semelhante ao de estudo realizado na cidade de Duque de Caxias- $R J^{(30)}$, onde esse fator de risco atingiu a frequência de $70,8 \%$, sendo prevalente também entre as mulheres.

Ao estudar os hábitos alimentares dos servidores neste estudo, verificou-se a elevada frequência de consumo de gorduras saturadas, e o baixo consumo de frutas, verduras, legumes e feijão em ambos os gêneros, corroborando com os dados da Pesquisa de Orçamentos Familiares 2008-2009, que mostra aspectos negativos sobre o padrão alimentar dos brasileiros, incluindo alto aporte de gorduras não saudáveis (gorduras saturadas e trans), baixo consumo de frutas, legumes e verduras, e alto consumo de alimentos com teores elevados de açúcar ${ }^{(31)}$.

Vários estudos vêm demonstrando associação entre escolhas alimentares inadequadas, como o consumo de gorduras saturadas e o baixo consumo de vegetais, e a incidência de excesso de peso e $\mathrm{DCNT}^{(30-32)}$.

Segundo dados da WHO, o consumo insuficiente de FLV é responsável anualmente por 2,7 milhões de mortes, $31 \%$ das doenças isquêmicas do coração, $11 \%$ das doenças cerebrovasculares e 19\% dos cânceres gastrointestinais ocorridos em todo o mundo ${ }^{(5)}$.

O consumo insuficiente de FLV neste estudo foi mais frequente entre as mulheres, diferindo dos achados em estudo de base populacional que investigou a prevalência de consumo de itens da dieta no Brasil ${ }^{(29)}$, o qual verificou que, entre os adultos, o consumo de FLV mais do que cinco dias na semana era menos frequente entre os homens $(12,9 \%)$ do que entre as mulheres $(26,9 \%)$. Porém, é semelhante aos encontrados em um estudo com trabalhadores de hotéis do litoral baiano, onde as mulheres tinham hábitos alimentares menos saudáveis, consumindo menos FLV do que os homens ${ }^{(32)}$. Em estudo com adultos na cidade de Belém$\mathrm{PA}^{(33)}$, verificou-se que os homens consumiam mais carne com gordura aparente do que as mulheres, no entanto, o consumo de leite integral era superior entre as mulheres. No presente estudo, tanto a frequência de consumo de carne com gordura aparente como de leite integral foi maior entre as mulheres. Os servidores nas faixas etárias de 18 a $24 \mathrm{e}$ maior que 65 anos consumiam menos leite com teor integral de gordura. Esses dados diferem dos encontrados na POF $(2008-2009)^{(31)}$, em que o maior consumo de leite integral foi entre os indivíduos jovens e idosos.

Alimentos como doces e refrigerantes são considerados menos saudáveis, pois sua ingestão ocasiona um alto consumo calórico ${ }^{(34,35)}$. Alguns estudos tentam buscar evidências de que o consumo excessivo de açúcares pode causar efeitos prejudiciais à saúde, todavia, a associação mais consistente tem sido entre uma alta ingestão de açúcar ou bebidas adoçadas e o desenvolvimento de obesidade ${ }^{(36,37)}$.

Neste estudo, o consumo de refrigerantes mais do que cinco dias na semana foi igual entre os gêneros, resultado diferente ao da pesquisa realizada sobre o consumo de refrigerantes com adultos de Pelotas-RS, na qual o consumo de refrigerantes foi maior entre os homens ${ }^{(30)}$.

O consumo insuficiente de feijão foi prevalente entre as mulheres, corroborando com os resultados de pesquisa nacional ${ }^{(14)}$. O consumo tendeu diminuir com o aumento do nível de escolaridade e idade.

Doenças como hipertensão, diabetes, obesidade e dislipidemias, concomitantes ou não, são fatores de risco para as doenças cardiovasculares, estando entre as principais causas de morte dos brasileiros ${ }^{(38)}$.

Em pesquisa realizada com servidores públicos das regiões Nordeste, Sudeste e Sul do Brasil ${ }^{(19)}$, verificouse que $36,1 \%$ referiram diagnostico prévio de HAS. No presente estudo, o percentual de diagnóstico prévio dessa morbidade foi de $25,8 \%$, com maior frequência entre as mulheres. Semelhante a outro estudo realizado em $2009^{(20)}$, que verificou maior frequência de HAS em mulheres.

As dislipidemias obtiveram frequência de $29,8 \%$, resultado menor do que o encontrado entre servidores de Fortaleza- $\mathrm{CE}^{(39)}$, onde em média $41,8 \%$ apresentavam dislipidemias, sendo estas mais prevalentes entre homens - o que difere dos servidores do presente estudo, já que a maior prevalência de dislipidemias foi entre mulheres, na faixa etária de 45 a 54 anos. É importante enfatizar que porcentagens de lipídios séricos podem aumentar com a idade em mulheres, devido à menopausa. Esta leva à diminuição da concentração de estrogênio, hormônio considerado protetor do sistema cardiovascular por sua ação de balanço do perfil lipídico, que auxilia na prevenção da aterosclerose ${ }^{(40)}$.

Os resultados do presente estudo concordam com outros estudos que têm demonstrado associação direta entre doenças crônicas e o aumento da idade ${ }^{(19,38,39)}$.

A maioria dos servidores, tendo cursado ensino superior, retrata um subgrupo da população que, em princípio, teria um melhor conhecimento sobre os riscos de uma alimentação inadequada, da prática insuficiente de atividade física, além de outros fatores de risco, pois 
teria maior acesso à informação e, com isso, seria menos exposto a esses fatores ${ }^{(10,19,39)}$, isto é, considerando que a escolaridade é um indicador da condição socioeconômica da população, e que altos níveis de escolaridade contribuem para uma melhor condição de vida e situação de saúde de grupos. Todavia, evidenciaram-se altas frequências de exposição aos fatores de risco entre os servidores, diferente de outros estudos, os quais demonstraram que existe uma maior associação de alguns desses fatores com a baixa escolaridade, principalmente entre as mulheres ${ }^{(3,7,18)}$.

Esses achados indicam a necessidade da realização de pesquisas sobre saúde e nutrição periodicamente, como também da elaboração e estruturação de programas de intervenções que visem à implementação de estratégias voltadas para a prevenção de DCNT e melhoria da qualidade de vida dos servidores avaliados.

Apesar de apresentar algumas limitações, devido ao delineamento transversal e à amostra por conveniência, o que dificultou a confirmação de certas tendências de associações entre as variáveis investigadas, este estudo é importante para esclarecer aspectos relacionados ao estilo de vida e fatores de risco para DCNT entre os servidores desta instituição pública de ensino superior da região Norte, cujas pesquisas ainda são limitadas no que se refere a questões relacionadas à alimentação, nutrição, qualidade de vida e de saúde dessa população.

\section{CONCLUSÃO}

Encontrou-se elevada prevalência de fatores de risco para doenças crônicas não transmissíveis na amostra estudada, com associação para sexo, faixa etária e escolaridade. $\mathrm{O}$ excesso de peso apresentou maior ocorrência nas mulheres e nas idades acima de 45 anos, hábitos alimentares inadequados no sexo feminino, maior ingestão de medida alcoólica no masculino e hábito de fumar nos indivíduos com maior escolaridade.

\section{REFERÊNCIAS}

1. Casado L, Viana LM, Thuler LCS. Fatores de risco para doenças crônicas não transmissíveis no Brasil: uma revisão sistemática. Rev Bras Cancerol. 2009;55(4):379-88.

2. Ministério da Saúde (BR), Secretaria de Vigilância em Saúde. Vigilância de fatores de risco e proteção para doenças crônicas por inquérito telefônico. Brasília: Ministério da Saúde; 2012.

3. Leitão MP, Martins IS. Prevalência e fatores associados a síndrome metabólica em usuários de Unidades Básicas de Saúde em São Paulo - SP. AMB Rev Assoc Med Bras. 2012;58(1):60-9.
4. World Health Organization - WHO. Noncommunicable diseases country profiles 2011. Geneva: WHO; 2011.

5. World Health Organization - WHO. Preventing chronic diseases: a vital investment: WHO global report. Geneva: WHO; 2010.

6. Instituto Brasileiro de Geografia e Estatística - IBGE. Pesquisa de orçamentos familiares pof 2008-2009: antropometria e análise do estado nutricional de crianças, adolescentes e adultos no Brasil. Rio de Janeiro: IBGE; 2010.

7 á NNB, Moura EC. Excesso de peso: determinantes sociodemográficos e comportamentais em adultos. Cad Saúde Pública. 2011;27(7):1380-92.

8. Ministério da Saúde (BR), Secretaria de Vigilância em Saúde, Secretaria de Atenção à Saúde. Política Nacional de Promoção da Saúde. Brasília: Ministério da Saúde; 2006. (Série B. Textos Básicos de Saúde).

9. Burity V, Franceschini T, Valente F, Recine E, Leão M, Carvalho MF. Direito humano à alimentação adequada no contexto da segurança alimentar e nutricional. Brasília: ABRANDH; 2010.

10. Malta DC, Iser BPM, Claro RM, Moura L, Bernal RTI, Nascimento AF, et al. Prevalência de fatores de risco e proteção para doenças crônicas não transmissíveis em adultos: estudo transversal, Brasil, 2011. Epidemiol Serv Saúde. 2013;22(3):423-34.

11. Lohman TG. Anthropometric standardization reference manual. Portland: Human Kinetics Pub; 1988.

12. Ministério da Saúde (BR). Vigilância alimentar e nutricional - Sisvan: orientações básicas para a coleta, processamento, análise de dados e informação em serviços de saúde. Brasília: Ministério da Saúde; 2004. (Série A. Normas e Manuais Técnicos)

13. World Health Organization - WHO. Obesity: preventing and managing the global epidemic. Report a WHO Consultation on Obesity. Geneva: WHO; 2000.

14. Ministério da Saúde (BR), Secretaria de Vigilância em Saúde, Departamento de Vigilância de Doenças e Agravos não Transmissíveis e Promoção de Saúde. VIGITEL Brasil 2012: vigilância de fatores de risco e proteção para doenças crônicas por inquérito telefônico. Brasília: Ministério da Saúde; 2013.

15. World Health Organization - WHO. Global recommendations on physical activity for health. Geneva: WHO; 2011.

16. Epi Info ${ }^{\mathrm{TM}}$ for DOS, 3.2.1 [software]. Atlanta: Centers for Disease Control and Prevention - Division of Public 
Health Surveillance and Informatics; 2004. Disponível em: http://www.cdc.gov/epiinfo/Epi6/ei6.htm

17. Ayres M, Ayres Junior M, Ayres DL, Santos AA. BIOESTAT Aplicações estatísticas nas áreas das ciências biomédicas [software]. Belém; 2007.

18. Malta DC. Doenças crônicas não transmissíveis, um grande desafio da sociedade contemporânea. Ciênc Saúde Coletiva. 2014;19(1):4

19. Duncan BB, Chor D, Aquino EML, Bensenor IM, Mill JG, Schimdt PAL, et al. Doenças crônicas não transmissíveis no Brasil: prioridade para enfrentamento e investigação. Rev Saúde Pública. 2012;46(Supl 1):126-34.

20. Oliveira LPM, Assis AMO, Silva MCM, Santana MLP, Santos NS, Pinheiro SMC, et al. Fatores associados a excesso de peso e concentração de gordura abdominal em adultos na cidade de Salvador, Bahia, Brasil. Cad Saúde Pública. 2009;25(3):570-82.

21. Lino MZR, Muniz PT, Siqueira KS. Prevalência e fatores associados ao excesso de peso em adultos: inquérito populacional em Rio Branco, Acre, Brasil, 2007-2008. Cad Saúde Pública. 2011;27(4):797-810

22. Laranjeira R, Pinsky I, Zaleski M, Caetano R. I Levantamento Nacional sobre os padrões de consumo de álcool na população brasileira. Brasília: Secretaria Nacional Antidrogas; 2007 [acesso em 2014 Fev 14]. Disponível em: http://bvsmssaude.gov.br/bvs/ publicacoes/relatorio_padroes_consumo_alcool.pdf

23. World Organization of Health - WHO. The newly published Global status report on alcohol and health analyses available evidence on alcohol consumption and provides data in $\mathrm{x}$ individual country profiles. 2011 [acesso em 2014 Fev 14]. Disponível em: http://www.who.int/mediacentre/news/releases/2011/ alcohol_20110211/en/

24. Aliança de Controle do Tabagismo. Carga das doenças tabaco-relacionadas para o Brasil [acesso em 2014 Mar 14]. Disponível em: http:// actbr.org.br/uploads/ conteudo/721_Relatorio_Carga_do_tabagismo_Brasil. pdf

25. Zamai CA, Bankoff ADP, Moraes MAA. Levantamento do índice de fatores de risco para doenças crônicas não transmissíveis entre servidores da UNICAMP. Conexões Rev Fac Educ Fís UNICAMP. 2012;10(1):115-41.

26. Conceição TV, Gomes FA, Tauil PL, Rosa TT. Valores de pressão arterial e suas associações com fatores de riscos cardiovasculares em servidores da Universidade de Brasília. Arq Bras Cardiol. 2006;86(1):26-31
27. Sousa AG, Carvalho CA, Almeida Fonseca PC, Machado SP. Perfil sociodemográfico e nutricional de servidores em período de pré-aposentadoria. Rev Pesq Saúde. 2011;12(3):16-21.

28. Nobre LN, Esteves EA, Silva KC, Moreira LL, Dias AM, Coelho NF, et al. Fatores de risco modificáveis para doenças cardiovasculares: efeito de um programa de educação. Alim Nutr (Araraquara). 2012;23(4):6719 .

29. Iser BPM, Yokota RTC, Sá NNB, Moura L, Malta DC. Prevalência de fatores de risco e proteção para doenças crônicas nas capitais do Brasil - principais resultados do VIGITEL 2010. Ciênc Saúde Coletiva. 2014;17(9):2343-56.

30. Rombaldi AJ, Neutzling MB, Silva MCD, Azevedo MR, Hallal PC. Factors associated with regular nondiet soft drink intake among adults in Pelotas, Southern Brazil. Rev Saúde Pública. 2011;45(2):382-90.

31. Instituto Brasileiro de Geografia e Estatística - IBGE. Pesquisa de orçamentos familiares 2008-2009: análise do consumo alimentar pessoal no Brasil / IBGE, Coordenação de Trabalho e Rendimento. Rio de Janeiro: IBGE; 2011.

32. Rocha CL, Garcia L. Avaliação nutricional, alimentar e dos hábitos de vida de trabalhadores de um hotel do litoral baiano. Rev Unifebe. 2012;10(1):59-67.

33. Neves ACM, Gonzaga LAA, Martens IBG, Moura EC. Validação de indicadores do consumo de alimentos e bebidas obtidos por inquérito telefônico em Belém, Pará, Brasil. Cad Saúde Pública. 2010;26(12):2379-88

34. Costa LC, Thuler LCS. Fatores associados ao risco para doenças não transmissíveis em adultos brasileiros. Rev Bras Estudos de Populações. 2012;29(1):133-45.

35. Flores TR, Ciochetto CR, Nunes BP, Vieira MFA. Consumo de refrigerantes entre escolares de séries iniciais da cidade de Pelotas, Rio Grande do Sul. Ciênc Saúde (Porto Alegre). 2013;6(1):59-66.

36. Gibson S. Sugar-sweetened soft drinks and obesity: a systematic review of the evidence from observational studies and interventions. Nutr Res Rev. 2008;21(2):134-47.

37. Forshee RA, Anderson PA, Storey ML. Sugarsweetened beverages and body mass index in children and adolescents: a meta-analysis. Am J Clin Nutr. 2008;87(6):1662-71. Errata em: Am J Clin Nutr. 2009;89(1):441-2.

38. Santos RD, Gagliardi ACM, Xavier HT, Magnoni CD, Cassani R, Lottenberg AMP, et al. I Diretriz sobre o 
consumo de gorduras e saúde cardiovascular. Arq Bras Cardiol. 2013;100(1Supl 3):1-49.

39. Amorim W, Oliveira RAR, Breguez MS, Amorim PRS, Marins, JCB. Prevalência de dislipidemia e sua relação com consumo de oxigênio entre servidores públicos. Rev Bras Promoç Saúde. 2013;26(2):290-7.
40. Oliveira A, Mancini Filho J. Perfil nutricional e lipídico de mulheres na pós-menopausa com doença arterial coronariana. Arq Bras Cardiol. 2005;84(4):325-9.

\section{Endereço para correspondência:}

Luciana Soares do Nascimento

Avenida Generalíssimo Deodoro, 01

Bairro: Umarizal

CEP: 66.050 .060 - Belém - Pará - Brasil

E-mail: 1sn.luciana27@gmail.com 